\title{
The global attractors and dimensions estimation for the Kirchhoff type wave equations with nonlinear strongly damped terms ${ }^{1}$ \\ Chengfei Ai, Huixian Zhu, Guoguang Lin² \\ Department of Mathematics, Yunnan University \\ Kunming, Yunnan 650091 \\ People's Republic of China
}

This paper studies the long time behavior of the solution to the initial boundary value problems for a class of nonlinear strongly damped Kirchhoff type wave equations:

$$
u_{t t}-\varepsilon_{1} \Delta u_{t}+\alpha\left|u_{t}\right|^{p-1} u_{t}+\beta|u|^{q-1} u-\phi\left(\mathrm{P} \nabla u \mathrm{P}^{2}\right) \Delta u=f(x) .
$$

Firstly, we prove the existence and uniqueness of the solution by priori estimate and the Galerkin method. Then we obtain to the existence of the global attractor. Finally, we consider that the estimation of the upper bounds of Hausdorff and fractal dimensions for the global attractor is obtained.

Key words: The nonlinear strong damping; Kirchhoff wave equation; The existence and uniqueness; Global attractor; Hausdorff dimension; Ftactal dimension

\section{Introduction}

It is well known that the dynamical systems that arise in physics, chemistry or biology, are often generated by a partial differential equation or a functional differential equation and thus the underlying state space is infinite-dimensional. The long time behavior of many dynamical systems generated by evolution equations can be described naturally in term of attractors of corresponding semigroups. The attractor is a basic concept in the study of the asymptotic behavior of solutions for the nonlinear evolution equations with various dissipation.

In recent years, many scholars have made useful researches for the existence of global attractors and their dimensions estimation about some the infinite dimension dynamic systems[1,2,3,4,5].

In this paper, we are concerned with the Kirchhoff type wave equations with nonlinear strongly damped terms referred to as follows:

$$
\begin{aligned}
& u_{t t}-\varepsilon_{1} \Delta u_{t}+\alpha\left|u_{t}\right|^{p-1} u_{t}+\beta|u|^{q-1} u-\phi\left(\mathrm{P} \nabla u \mathrm{P}^{2}\right) \Delta u=f(x) \quad \text { in } \Omega \times \mathrm{R}^{+} \\
& u(x, 0)=u_{0}(x) ; u_{t}(x, 0)=u_{1}(x), \quad x \in \Omega \\
& \left.u(x, t)\right|_{\partial \Omega}=0,\left.\Delta u(x, t)\right|_{\partial \Omega}=0, \quad x \in \Omega .
\end{aligned}
$$

where $\Omega$ is a bounded domain in $\mathrm{R}^{N}$ with smooth boundary $\partial \Omega$, and $\varepsilon_{1}, \alpha, \beta$ are positive constants, and the assumptions on $\phi\left(\mathrm{P} \Delta u \mathrm{P}^{2}\right)$ will be specified later.

In [6], G. Kirchhoff firstly proposed the so called Kirchhoff string model in the study nonlinear vibration of an elastic string:

$$
\rho h u_{t t}+\delta u_{t}=p_{0}+\frac{E h}{2 L}\left(\int_{0}^{L}\left|u_{x}\right|^{2} d x\right) u_{x x}+f(x), 0<x<L, t>0
$$

where $u=u(x, t)$ is the lateral displacement at the space coordinate $x$ and the time $t, E$ is the Young modules, $h$ is the cross-section area, $\rho$ is the mass density, $L$ is the length, $p_{0}$ is the initial axial tension, $\delta$ is the resistance modules and $f$ is the external force.

Yang Zhijian, Ding Pengyan and Liu Zhiming [7] studied the Global attractor for the Kirchhoff type equations with strong nonlinear damping and supercritical nonlinearity:

$$
u_{t t}-\sigma\left(\mathrm{P} \Delta u \mathrm{P}^{2}\right) \Delta u_{t}-\phi\left(\mathrm{P} \Delta u \mathrm{P}^{2}\right) \Delta u+f(u)=h(x) \text { in } \Omega \times \mathrm{R}^{+}
$$




$$
\left.u(x, t)\right|_{\partial \Omega}=0, u(x, 0)=u_{0}(x), u_{t}(x, 0)=u_{1}(x), \quad x \in \Omega
$$

where $\Omega$ is a bounded domain in $R^{N}$ with the smooth boundary $\partial \Omega, \sigma(s), \phi(s)$ and $f(s)$ are nonlinear functions, and $h(x)$ is an external force term.

Yang Zhijian, Wang Yunqing [8] also studied the global attractor for the Kirchhoff type equation with a strong dissipation:

$$
\begin{aligned}
& u_{t t}-M\left(\mathrm{P} \Delta u \mathrm{P}^{2}\right) \Delta u-\Delta u_{t}+h\left(u_{t}\right)+g(u)=f(x) \text { in } \Omega \times \mathrm{R}^{+}, \\
& \left.u(x, t)\right|_{\partial \Omega}=0, t>0, u(x, 0)=u_{0}(x), u_{t}(x, 0)=u_{1}(x), \quad x \in \Omega .
\end{aligned}
$$

where $M(s)=1+s^{\frac{m}{2}}, 1 \leq m \leq \frac{4}{(N-2)}, \Omega$ is a bounded domain in $R^{N}$, with smooth boundary $\partial \Omega, h(s)$ and $g(s)$ are nonlinear functions, and $f(x)$ is an external force term.

Recently, Meixia Wang, Cuicui Tian, Guoguang Lin [9] studied the global attractor and dimension estimation for a 2D generalized Anisotropy Kuramoto-Sivashinsky equation:

$$
\begin{aligned}
& u_{t}+\alpha \Delta^{2} u+\gamma+(\varphi(u))_{x x}+(g(u))_{y y}=f(x),(x, y) \in \Omega \subset R^{2}, \\
& \left.u(x, y, t)\right|_{t=0}=u_{0}(x, y),(x, y) \in \Omega \subset R^{2} \\
& \left.u(x, y, t)\right|_{\partial \Omega=0}=0,\left.\Delta u(x, y, t)\right|_{\partial \Omega}=0,(x, y) \in \Omega \subset R^{2} .
\end{aligned}
$$

where $\Omega \subset R^{2}$ is bounded set; $\partial \Omega$ is the bound of $\Omega ; \varphi(u)$ and $g(u)$ are considered as smooth functions of $u(x, y, t)$.

There have been many researches on the long-time behavior of solutions to the nonlinear damped wave equations with delays. For more related results we refer the reader to [10]-[13]. In order to make these equations more normal, in section 2 and in section 3 , some assumptions, notations and the main results are stated. Under these assumptions, we prove the existence and uniqueness of solution, then we obtain the global attractors for the problems (1.1)-(1.3). According to [9][13], in section 4, we consider that the global attractor of the above mentioned problems (1.1)-(1.3) has finite Hausdorff dimensions and fractal dimensions.

\section{Statement of main results}

For convenience, we denote the norm and scalar product in $L^{2}(\Omega)$ by P.P and (.,.);

$$
f=f(x), L^{p}=L^{p}(\Omega), H^{k}=H^{k}(\Omega), H_{0}^{k}=H_{0}^{k}(\Omega), \mathrm{P} \cdot \mathrm{P}=\mathrm{P} \cdot \mathrm{P}_{L^{2}}, \mathrm{P} \cdot \mathrm{P}_{p}=\mathrm{P} \cdot \mathrm{P}{ }_{L^{p}} .
$$

In this section, we present some assumptions and notations needed in the proof of our results. For this reason, we assume that

$\left(G_{1}\right) \phi\left(\mathrm{P} \nabla u \mathrm{P}^{2}\right): R^{+} \rightarrow R^{+}$is a differentiable function;

$\left(G_{2}\right) \quad$ There exist constant $\quad \varepsilon_{1}>0, \varepsilon>0, \gamma_{1}>0, \gamma_{2}>0, K \geq 0$, such that $K-2 \varepsilon \geq 0$, $\varepsilon_{1} \varepsilon \leq \phi\left(\mathrm{P} \nabla u \mathrm{P}^{2}\right) \leq \frac{\gamma_{1}}{K-2 \varepsilon}\left(1+\gamma_{2} e^{-(K-2 \varepsilon) t}\right)$.

Lemma 1. Assume $\left(G_{1}\right),\left(G_{2}\right)$ hold, and $\left(u_{0}, u_{1}\right) \in\left(L^{q+1}(\Omega) \cap H_{0}^{1}(\Omega)\right) \times L^{2}(\Omega), f \in L^{2}(\Omega), v=u_{t}+\varepsilon u$, let

$$
\begin{cases}p \geq 2, & n=1,2 \\ 2<p<\frac{n+4}{n}, & n \geq 3 .\end{cases}
$$




$$
\begin{cases}q \geq 2, & n=1,2 \\ 2<q<\frac{n+2}{n-2}, & n \geq 3\end{cases}
$$

Then the solution $(u, v)$ of the problems $\quad(1.1)-(1.3)$ satisfies $(u, v) \in\left(L^{q+1}(\Omega) \cap H_{0}^{1}(\Omega)\right) \times L^{2}(\Omega), H_{1}:=L^{q+1}(\Omega) \cap H_{0}^{1}(\Omega)$, and

$$
\mathrm{P}(u, v) \mathrm{P}_{\mathrm{H}_{1} \times L^{2}}^{2}=\mathrm{P} \nabla u \mathrm{P}^{2}+\mathrm{P}_{v} \mathrm{P}^{2} \leq \frac{W(0)}{N} \mathrm{e}^{-\alpha_{1} t}+\frac{C}{N \alpha_{1}}\left(1-\mathrm{e}^{-\alpha_{1} t}\right) .
$$

where $v=u_{t}+\varepsilon u, \quad 0<N<\min \left\{1, \phi\left(\mathrm{P} \nabla u \mathrm{P}^{2}\right)-\varepsilon_{1} \varepsilon\right\}$, and $W(0)=\mathrm{P} v_{0} \mathrm{P}^{2}+\left(\phi\left(\mathrm{P} \nabla u_{0} \mathrm{P}^{2}\right)-\varepsilon_{1} \varepsilon\right) \mathrm{P} \nabla u_{0} \mathrm{P}^{2}$, $v_{0}=u_{1}+\varepsilon u_{0}$, thus there exist $R_{0}$ and $t_{1}=t_{1}(\Omega)>0$, such that

$$
\mathrm{P}(u, v) \mathrm{P}_{\mathrm{H}_{1} \times L^{2}}^{2}=\mathrm{P} \nabla u \mathrm{P}^{2}+\mathrm{P} v_{v} \mathrm{P}^{2} \leq R_{0}\left(t>t_{1}\right) .
$$

Proof. Let $v=u_{t}+\varepsilon u$, We multiply $v$ with both sides of equation (1.1) and obtain

$$
\left(u_{t t}-\varepsilon_{1} \Delta u_{t}+\alpha\left|u_{t}\right|^{p-1} u_{t}+\beta|u|^{q-1} u-\phi\left(\mathrm{P} \nabla u \mathrm{P}^{2}\right) \Delta u, v\right)=(f(x), v) .
$$

By using $\mathrm{H} \ddot{o}$ Ider's inequality, Young's inequality and Poincar $e^{\prime}$ 's inequality. one by one as follows:

$$
\begin{aligned}
& \left(u_{t t}, v\right)=\frac{1}{2} \frac{d}{d t} \mathrm{P} v \mathrm{P}^{2}-\varepsilon(v-\varepsilon u, v) \geq \frac{1}{2} \frac{d}{d t} \mathrm{P} v \mathrm{P}^{2}-\varepsilon \mathrm{P} v \mathrm{P}^{2}-\frac{\varepsilon^{2}}{2 \lambda_{1}} \mathrm{P} \nabla u \mathrm{P}^{2}-\frac{\varepsilon^{2}}{2} \mathrm{P} v \mathrm{P}^{2} \\
& \left(-\varepsilon_{1} \Delta u_{t}, v\right)=-\varepsilon_{1}(\Delta(v-\varepsilon u), v) \geq \varepsilon_{1} \lambda_{2} \mathrm{P} v \mathrm{P}^{2}-\frac{\varepsilon_{1} \varepsilon}{2} \frac{d}{d t} \mathrm{P} \nabla u \mathrm{P}^{2}-\varepsilon_{1} \varepsilon^{2} \mathrm{P} \nabla u \mathrm{P}^{2} \\
& \left(\alpha\left|u_{t}\right|^{p-1} u_{t}, v\right) \\
& =\alpha\left(\left|u_{t}\right|^{p-1} u_{t}, u_{t}+\varepsilon u\right) \\
& =\alpha \mathrm{P} u_{t} \mathrm{P}_{p+1}^{p+1}+\alpha \varepsilon \int_{\Omega}\left|u_{t}\right|^{p-1} u_{t} \cdot u d x
\end{aligned}
$$

where

$$
\begin{aligned}
& \alpha \varepsilon \int_{\Omega}\left|u_{t}\right|^{p-1} u_{t} \cdot u d x \\
& \leq \alpha \varepsilon \int_{\Omega}\left|u_{t}\right|^{p} \cdot|u| d x \\
& \leq \alpha \varepsilon\left(\int_{\Omega}\left|u_{t}\right|^{p+1} d x\right)^{\frac{p}{p+1}} \cdot\left(\int_{\Omega}|u|^{p+1} d x\right)^{\frac{1}{p+1}} \\
& =\alpha \varepsilon u_{t} \mathrm{P}_{p+1}^{p} \cdot \mathrm{P}_{u} \mathrm{P}_{p+1} \\
& \leq \frac{\alpha p}{p+1} \mathrm{P}_{t} \mathrm{P}_{p+1}^{p+1}+\frac{\alpha \varepsilon^{p+1}}{p+1} \mathrm{P} u \mathrm{P}_{p+1}^{p+1}
\end{aligned}
$$

And using Interpolation Theorem, we have

$$
\begin{aligned}
& \frac{\alpha p}{p+1} \mathrm{P} u_{t} \mathrm{P}_{p+1}^{p+1}+\frac{\alpha \varepsilon^{p+1}}{p+1} \mathrm{P} u \mathrm{P}_{p+1}^{p+1} \\
& \leq \frac{\alpha p}{p+1} \mathrm{P} u_{t} \mathrm{P}_{p+1}^{p+1}+C_{0}(\alpha, \varepsilon, p, \mathrm{P} u \mathrm{P}) \mathrm{P} \nabla u \mathrm{P}^{\frac{n(p-1)}{2}}
\end{aligned}
$$

then from $2<p<\frac{n+4}{n}, n \geq 3$, according to Embedding Theorem 


$$
\begin{aligned}
& \frac{\alpha p}{p+1} \mathrm{P} u_{t} \mathrm{P}_{p+1}^{p+1}+C_{0}(\alpha, \varepsilon, p, \mathrm{P} u \mathrm{P}) \mathrm{P} \nabla u \mathrm{P}^{\frac{n(p-1)}{2}} \\
& \leq \frac{\alpha p}{p+1} \mathrm{P}_{u_{t}} \mathrm{P}_{p+1}^{p+1}+\frac{\varepsilon_{1} \varepsilon^{2}}{2} \mathrm{P} \nabla u \mathrm{P}^{2}+C_{1}\left(\alpha, \varepsilon, p, \mathrm{P} u \mathrm{P}, \varepsilon_{1}\right) .
\end{aligned}
$$

and

$$
\begin{aligned}
& \left(\beta|u|^{q-1} u, v\right)=\frac{\beta}{(q+1)} \frac{d}{d t} \mathrm{P}_{u} \mathrm{P}_{q+1}^{q+1}+\beta \varepsilon \mathrm{P}_{u} \mathrm{P}_{q+1}^{q+1} \\
& \left(-\phi\left(\mathrm{P} \nabla u \mathrm{P}^{2}\right) \Delta u, v\right) \\
& =\phi\left(\mathrm{P} \nabla u \mathrm{P}^{2}\right) \frac{1}{2} \frac{d}{d t} \mathrm{P} \nabla u \mathrm{P}^{2}+\varepsilon \phi\left(\mathrm{P} \nabla u \mathrm{P}^{2}\right) \mathrm{P} \nabla u \mathrm{P}^{2} \\
& =\frac{d}{d t}\left[\frac{1}{2} \phi\left(\mathrm{P}^{2} u \mathrm{P}^{2}\right) \mathrm{P} \nabla u \mathrm{P}^{2}\right]-\frac{1}{2} \mathrm{P} \nabla u \mathrm{P}^{2} \frac{d}{d t}\left(\phi\left(\mathrm{P} \nabla u \mathrm{P}^{2}\right)\right)+\varepsilon \phi\left(\mathrm{P} \nabla u \mathrm{P}^{2}\right) \mathrm{P} \nabla u \mathrm{P}^{2} \\
& (f(x), v) \quad \leq \mathrm{P} f \mathrm{PP} v \mathrm{P} \leq \frac{\varepsilon^{2}}{2} \mathrm{P}_{v} \mathrm{P}^{2}+\frac{1}{2 \varepsilon^{2}} \mathrm{P}_{f} \mathrm{P}^{2} .
\end{aligned}
$$

From the above, we have

$$
\begin{aligned}
& \frac{d}{d t}\left[\mathrm{P} v \mathrm{P}^{2}+\left(\phi\left(\mathrm{P} \nabla u \mathrm{P}^{2}\right)-\varepsilon_{1} \varepsilon\right) \mathrm{P} \nabla u \mathrm{P}^{2}+\frac{2 \beta}{q+1} \mathrm{P}_{u} \mathrm{P}_{q+1}^{q+1}\right]+\left(2 \varepsilon_{1} \lambda_{2}-2 \varepsilon-2 \varepsilon^{2}\right) \mathrm{P}_{v} \mathrm{P}^{2} \\
& +\left[2 \varepsilon \phi\left(\mathrm{P} \nabla u \mathrm{P}^{2}\right)-\frac{d}{d t}\left(\phi\left(\mathrm{P} \nabla u \mathrm{P}^{2}\right)\right)-\frac{\varepsilon^{2}}{\lambda_{1}}-3 \varepsilon_{1} \varepsilon^{2}\right] \mathrm{P} \nabla u \mathrm{P}^{2}+2 \beta \varepsilon \mathrm{P}_{1} \mathrm{P}_{q+1}^{q+1}+ \\
& \frac{2 \alpha}{p+1} \mathrm{P}_{t} \mathrm{P}_{p+1}^{p+1} \leq \frac{1}{\varepsilon^{2}} \mathrm{P} f \mathrm{P}^{2}+2 C_{1}\left(\alpha, \varepsilon, p, \mathrm{P} u \mathrm{P}, \varepsilon_{1}\right):=C .
\end{aligned}
$$

Next, we take proper $\varepsilon, \varepsilon_{1}$, such that: $\varepsilon_{1} \varepsilon \leq \phi\left(\mathrm{P} \nabla u \mathrm{P}^{2}\right)$,

let constant $\mathrm{K}$, such that $K-2 \varepsilon \geq 0$,

$$
0 \leq K\left(\phi\left(\mathrm{P} \nabla u \mathrm{P}^{2}\right)-\varepsilon_{1} \varepsilon\right) \leq 2 \varepsilon \phi\left(\mathrm{P} \nabla u \mathrm{P}^{2}\right)-\frac{d}{d t}\left(\phi\left(\mathrm{P} \nabla u \mathrm{P}^{2}\right)\right)-\frac{\varepsilon^{2}}{\lambda_{1}}-3 \varepsilon_{1} \varepsilon^{2}
$$

Where $\left.C_{3}=C_{3}\left(\varepsilon, \lambda_{1}, \varepsilon_{1}\right)=\frac{\varepsilon^{2}}{\lambda_{1}}+3 \varepsilon_{1} \varepsilon^{2} £\right\urcorner$ such that

$$
\begin{aligned}
& 0 \leq K\left(\phi\left(\mathrm{P} \nabla u \mathrm{P}^{2}\right)-\varepsilon_{1} \varepsilon\right) \leq 2 \varepsilon \phi\left(\mathrm{P} \nabla u \mathrm{P}^{2}\right)-\frac{d}{d t}\left(\phi\left(\mathrm{P} \nabla u \mathrm{P}^{2}\right)\right)-C_{3} \\
& (K-2 \varepsilon) \phi\left(\mathrm{P} \nabla u \mathrm{P}^{2}\right)+\frac{d}{d t}\left(\phi\left(\mathrm{P} \nabla u \mathrm{P}^{2}\right)\right) \leq K \varepsilon_{1} \varepsilon-C_{3}:=\gamma_{1}
\end{aligned}
$$

Multiplying(2.16) by $e^{(K-2 \varepsilon) t}$, then

$$
\phi\left(\mathrm{P} \nabla u \mathrm{P}^{2}\right) \frac{d}{d t}\left(e^{(K-2 \varepsilon) t}\right)+e^{(K-2 \varepsilon) t} \frac{d}{d t}\left(\phi\left(\mathrm{P} \nabla u \mathrm{P}^{2}\right)\right) \leq \gamma_{1} e^{(K-2 \varepsilon) t},
$$

We integrate (2.17)with respect to time $t$ and get that

$$
\phi\left(\mathrm{P} \nabla u \mathrm{P}^{2}\right) \leq \frac{\gamma_{1}}{K-2 \varepsilon}\left(1+\gamma_{2} e^{-(K-2 \varepsilon) t}\right) .
$$


Where $\varepsilon, \gamma_{1}, \gamma_{2}$ is constant. From the above, we obtain

$$
\varepsilon_{1} \varepsilon \leq \phi\left(\mathrm{P} \nabla u \mathrm{P}^{2}\right) \leq \frac{\gamma_{1}}{K-2 \varepsilon}\left(1+\gamma_{2} e^{-(K-2 \varepsilon) t}\right)
$$

At last, we take proper $\varepsilon, \varepsilon_{1}, \beta$, such that:

$$
\left\{\begin{array}{l}
a_{1}=2 \varepsilon_{1} \lambda_{2}-2 \varepsilon-2 \varepsilon^{2} \geq 0 \\
a_{2}=2 \varepsilon \phi\left(\mathrm{P} \nabla u \mathrm{P}^{2}\right)-\frac{d}{d t}\left(\phi\left(\mathrm{P} \nabla u \mathrm{P}^{2}\right)\right)-\frac{\varepsilon^{2}}{\lambda_{1}}-3 \varepsilon_{1} \varepsilon^{2} \geq 0 .
\end{array}\right.
$$

Taking $\alpha_{1}=\min \left\{a_{1}, \frac{a_{2}}{\phi\left(\mathrm{P} \nabla u \mathrm{P}^{2}\right)-\varepsilon_{1} \varepsilon}, \varepsilon(q+1)\right\}$, then

$$
\frac{d}{d t} W(t)+\alpha_{1} W(t) \leq C
$$

Where $W(t)=\mathrm{P}_{v} \mathrm{P}^{2}+\left(\phi\left(\mathrm{P} \nabla u \mathrm{P}^{2}\right)-\varepsilon_{1} \varepsilon\right) \mathrm{P} \nabla u \mathrm{P}^{2}+\frac{2 \beta}{q+1} \mathrm{P} u \mathrm{P}_{q+1}^{q+1}$, by using Gronwall's inequality, we obtain:

$$
W(t) \leq W_{0} e^{-\alpha_{1} t}+\frac{C}{\alpha_{1}}\left(1-\mathrm{e}^{-\alpha_{1} t}\right)
$$

From $2<q<\frac{n+2}{n-2}, n \geq 3$, according to Embedding Theorem $H_{0}^{1}(\Omega)^{\circ} L^{q+1}(\Omega)$, let $0<N=\min \left\{1, \phi\left(\mathrm{P} \nabla u \mathrm{P}^{2}\right)-\varepsilon_{1} \varepsilon\right\}$, such that:

$$
\mathrm{P}(u, v) \mathrm{P}_{\mathrm{H}_{1} \times L^{2}}^{2}=\mathrm{P} \nabla u \mathrm{P}^{2}+\mathrm{P}_{v} \mathrm{P}^{2} \leq \frac{W(0)}{N} \mathrm{e}^{-\alpha_{1} t}+\frac{C}{N \alpha_{1}}\left(1-\mathrm{e}^{-\alpha_{1} t}\right) .
$$

where $v=u_{t}+\varepsilon u$, and $W(0)=\mathrm{P} v_{0} \mathrm{P}^{2}+\left(\phi\left(\mathrm{P} \nabla u_{0} \mathrm{P}^{2}\right)-\varepsilon_{1} \varepsilon\right) \mathrm{P} \nabla u_{0} \mathrm{P}^{2}+\frac{\beta}{q+1} \mathrm{P} u_{0} \mathrm{P}_{q+1}^{q+1}, v_{0}=u_{1}+\varepsilon u_{0}$, then

$$
\varlimsup_{t \rightarrow \infty} \mathrm{P}(u, v) \mathrm{P}_{\mathrm{H}_{1} \times L^{2}}^{2} \leq \frac{C}{N \alpha_{1}},
$$

thus there exist $R_{0}$ and $t_{1}=t_{1}(\Omega)>0$, such that

$$
\mathrm{P}(u, v) \mathrm{P}_{\mathrm{H}_{1} \times L^{2}}^{2}=\mathrm{P} \nabla u \mathrm{P}^{2}+\mathrm{P}_{v} \mathrm{P}^{2} \leq R_{0}\left(t>t_{1}\right) .
$$

Lemma 2. In addition to the assumptions of Lemma 1, $\left(G_{1}\right),\left(G_{2}\right)$ hold.

If $\left(G_{3}\right): f \in H_{0}^{1}(\Omega)$, let

$$
\begin{aligned}
& \begin{cases}p \geq 2, & n=1,2 ; \\
2<p<\frac{n+4}{n}, & n \geq 3 .\end{cases} \\
& \begin{cases}q \geq 2, & n=1,2 ; \\
2<q<\frac{n+4}{n}, & n \geq 3 .\end{cases}
\end{aligned}
$$


and $\left(u_{0}, u_{1}\right) \in\left(H^{2}(\Omega) \cap H_{0}^{1}(\Omega)\right) \times H_{0}^{1}(\Omega), v=u_{t}+\varepsilon u$, Then the solution $(u, v)$ of the problems (1.1)-(1.3) satisfies $(u, v) \in\left(H^{2}(\Omega) \cap H_{0}^{1}(\Omega)\right) \times H_{0}^{1}(\Omega), \mathrm{H}_{2}:=H^{2}(\Omega) \cap H_{0}^{1}(\Omega)$, and

$$
\mathrm{P}(u, v) \mathrm{P}_{\mathrm{H}_{2} \times H_{0}^{1}}^{2}=\mathrm{P} \Delta u \mathrm{P}^{2}+\mathrm{P} \nabla v \mathrm{P}^{2} \leq \frac{U(0)}{M} \mathrm{e}^{-\alpha_{2} t}+\frac{C_{1}}{M \alpha_{2}}\left(1-\mathrm{e}^{-\alpha_{2} t}\right) .
$$

where

$$
v=u_{t}+\varepsilon u
$$

$0<M<\min \left\{1, \phi\left(\mathrm{P} \nabla u \mathrm{P}^{2}\right)+\varepsilon_{1} \varepsilon\right\}$,

$U(0)=\mathrm{P} \nabla v_{0} \mathrm{P}^{2}+\left(\phi\left(\mathrm{P} \nabla u_{0} \mathrm{P}^{2}\right)+\varepsilon_{1} \varepsilon\right) \mathrm{P} \Delta u_{0} \mathrm{P}^{2}$, thus there exist $R_{1}$ and $t_{2}=t_{2}(\Omega)>0$, such that

$$
\mathrm{P}(u, v) \mathrm{P}_{\mathrm{H}_{2} \times H_{0}^{1}}^{2}=\mathrm{P} \Delta u \mathrm{P}^{2}+\mathrm{P} \nabla v \mathrm{P}^{2} \leq R_{1}\left(t>t_{2}\right) .
$$

Proof. Let $-\Delta v=-\Delta u_{t}-\varepsilon \Delta u$, We multiply $-\Delta v$ with both sides of equation (1.1) and obtain

$$
\left(u_{t t}-\varepsilon_{1} \Delta u_{t}+\alpha\left|u_{t}\right|^{p-1} u_{t}+\beta|u|^{q-1} u-\phi\left(\mathrm{P} \nabla u \mathrm{P}^{2}\right) \Delta u,-\Delta v\right)=(f(x),-\Delta v) .
$$

By using $\mathrm{H} \ddot{o}$ Ider's inequality, Young's inequality and Poincar $e^{\prime}$ 's inequality, we deal with the terms in (2.27), one by one as follows:

$$
\begin{aligned}
& \left(u_{t t},-\Delta v\right) \geq \frac{1}{2} \frac{d}{d t} \mathrm{P} \nabla v \mathrm{P}^{2}-\varepsilon \mathrm{P} \nabla v \mathrm{P}^{2}-\frac{\varepsilon^{2}}{2 \mu_{1}} \mathrm{P} \Delta u \mathrm{P}^{2}-\frac{\varepsilon^{2}}{2} \mathrm{P} \nabla v \mathrm{P}^{2} . \\
& \left(-\varepsilon_{1} \Delta u_{t},-\Delta v\right) \\
& \quad=\left(-\varepsilon_{1} \Delta u_{t},-\Delta u_{t}-\varepsilon \Delta u\right) \\
& \quad=\varepsilon_{1} \mathrm{P} \Delta u_{t} \mathrm{P}^{2}+\varepsilon_{1} \mathrm{P} \Delta v \mathrm{P}^{2}-\varepsilon_{1}\left(\Delta v, \Delta u_{t}\right)-\varepsilon_{1} \varepsilon(\Delta u, \Delta v)+\varepsilon_{1} \varepsilon\left(\Delta u, \Delta u_{t}\right) \\
& \quad \geq \frac{\varepsilon_{1}-\varepsilon_{1} \varepsilon}{2} \mathrm{P} \Delta v \mathrm{P}^{2}+\frac{\varepsilon_{1}}{2} \mathrm{P} \Delta u_{t} \mathrm{P}^{2}+\frac{\varepsilon_{1} \varepsilon}{2} \frac{d}{d t} \mathrm{P} \Delta u \mathrm{P}^{2}-\frac{\varepsilon_{1} \varepsilon}{2} \mathrm{P} \Delta u \mathrm{P}^{2} . \\
& \left(\alpha\left|u_{t}\right|^{p-1} u_{t},-\Delta v\right)=\alpha\left(\left|u_{t}\right|^{p-1} u_{t},-\Delta u_{t}\right)+\alpha\left(\left|u_{t}\right|^{p-1} u_{t},-\varepsilon \Delta u\right) .
\end{aligned}
$$

Where

$$
\begin{gathered}
\alpha\left(\left|u_{t}\right|^{p-1} u_{t},-\Delta u_{t}\right) \\
=\alpha\left(\nabla\left(\left(u_{t}^{2}\right)^{\frac{p-1}{2}} u_{t}\right), \nabla u_{t}\right) \\
=\alpha\left(\frac{p-1}{2}\left(u_{t}^{2}\right)^{\frac{p-3}{2}} 2 u_{t}^{2} \nabla u_{t}, \nabla u_{t}\right)+\alpha\left(\left|u_{t}\right|^{p-1} \nabla u_{t}, \nabla u_{t}\right) \\
=\alpha(p-1)\left(\left|u_{t}\right|^{p-1} \nabla u_{t}, \nabla u_{t}\right)+\alpha\left(\left|u_{t}\right|^{p-1} \nabla u_{t}, \nabla u_{t}\right) \\
=\alpha p \int_{\Omega}\left|u_{t}\right|^{p-1}\left|\nabla u_{t}\right|^{2} d x>0, \\
\alpha\left(\left|u_{t}\right|^{p-1} u_{t},-\varepsilon \Delta u\right) \quad \leq \alpha \varepsilon \int_{\Omega}\left|u_{t}\right|^{p}|\Delta u| d x \\
\quad \leq \alpha \varepsilon\left(\int_{\Omega}\left|u_{t}\right|^{2 p} d x\right)^{\frac{1}{2}}\left(\int_{\Omega}|\Delta u|^{2} d x\right)^{\frac{1}{2}} \\
\quad=\alpha \varepsilon u_{t} \mathrm{P}_{2 p}^{p} \mathrm{P} \Delta u \mathrm{P},
\end{gathered}
$$

By using Interpolation Theorem and Embedding Theorem: 


$$
\begin{aligned}
\alpha \varepsilon \mathrm{P} u_{t} \mathrm{P}_{2 p}^{p} \mathrm{P} \Delta u \mathrm{P} & \leq \alpha \varepsilon C_{0}\left(\mathrm{P} u_{t} \mathrm{P}\right) \mathrm{P} \Delta u_{t} \mathrm{P}^{\frac{n(p-1)}{4}} \mathrm{P} \Delta u \mathrm{P} \\
& \leq \frac{\varepsilon^{2}}{2 \mu_{1}} \mathrm{P} \Delta u \mathrm{P}^{2}+\frac{\mu_{1} \alpha^{2} C_{0}^{2}\left(\mathrm{P} u_{t} \mathrm{P}\right)}{2} \mathrm{P} \Delta u_{t} \mathrm{P}^{\frac{n(p-1)}{2}} \\
& \leq \frac{\varepsilon^{2}}{2 \mu_{1}} \mathrm{P} \Delta u \mathrm{P}^{2}+\frac{\varepsilon_{1}}{4} \mathrm{P} \Delta u_{t} \mathrm{P}^{2}+C_{1}\left(\alpha, C_{0}, \mu_{1}, \varepsilon_{1}\right),
\end{aligned}
$$

Substituting (2.31),(2.32),(2.33) into (2.30), we receive

$$
\begin{aligned}
& \left(\alpha\left|u_{t}\right|^{p-1} u_{t},-\Delta v\right) \\
& \geq \alpha p \int_{\Omega}\left|u_{t}\right|^{p-1}\left|\nabla u_{t}\right|^{2} d x-\frac{\varepsilon^{2}}{2 \mu_{1}} \mathrm{P} \Delta u \mathrm{P}^{2}-\frac{\varepsilon_{1}}{4} \mathrm{P} \Delta u_{t} \mathrm{P}^{2}-C_{1} .
\end{aligned}
$$

By using Interpolation Theorem and Embedding Theorem:

$$
\begin{aligned}
& \left(\beta|u|^{q-1} u,-\Delta v\right) \\
& \leq \beta \mathrm{P} u \mathrm{P}_{2 q}^{q} \mathrm{P} \Delta v \mathrm{P} \\
& \leq \beta C_{2}(\mathrm{P} u \mathrm{P}) \mathrm{P} \Delta u \mathrm{P}^{\frac{n(q-1)}{4}} \mathrm{P} \Delta v \mathrm{P} \\
& \leq \frac{\varepsilon_{1}-\varepsilon_{1} \varepsilon}{4} \mathrm{P} \Delta v \mathrm{P}^{2}+\frac{\beta^{2} C_{2}^{2}(\mathrm{P} u \mathrm{P})}{\varepsilon_{1}-\varepsilon_{1} \varepsilon} \mathrm{P} \Delta u \mathrm{P}^{\frac{n(q-1)}{2}} \\
& \leq \frac{\varepsilon_{1}-\varepsilon_{1} \varepsilon}{4} \mathrm{P} \Delta \nu \mathrm{P}^{2}+\frac{\varepsilon_{1} \varepsilon}{2} \mathrm{P} \Delta u \mathrm{P}^{2}+C_{3}\left(\varepsilon, \beta, \varepsilon_{1}, \mathrm{P} u \mathrm{P}\right) \text {. } \\
& \left(-\phi\left(\mathrm{P} \nabla u \mathrm{P}^{2}\right) \Delta u,-\Delta v\right) \\
& =\phi\left(\mathrm{P} \nabla u \mathrm{P}^{2}\right)\left(\Delta u, \Delta u_{t}+\varepsilon \Delta u\right) \\
& =\frac{1}{2} \phi\left(\mathrm{P} \nabla u \mathrm{P}^{2}\right) \frac{d}{d t} \mathrm{P} \Delta u \mathrm{P}^{2}+\varepsilon \phi\left(\mathrm{P} \nabla u \mathrm{P}^{2}\right) \mathrm{P} \Delta u \mathrm{P}^{2} \\
& =\frac{d}{d t}\left[\frac{1}{2} \phi\left(\mathrm{P} \nabla u \mathrm{P}^{2}\right) \mathrm{P} \Delta u \mathrm{P}^{2}\right]-\frac{1}{2} \mathrm{P} \Delta u \mathrm{P}^{2} \frac{d}{d t}\left(\phi\left(\mathrm{P} \nabla u \mathrm{P}^{2}\right)\right)+\varepsilon \phi\left(\mathrm{P} \nabla u \mathrm{P}^{2}\right) \mathrm{P} \Delta u \mathrm{P}^{2} . \\
& (f,-\Delta v) \leq \mathrm{P} \nabla f \mathrm{PP} \nabla v \mathrm{P} \leq \frac{\varepsilon_{1}-\varepsilon_{1} \varepsilon}{8 \mu_{2}} \mathrm{P} \nabla v \mathrm{P}^{2}+\frac{2 \mu_{2}}{\varepsilon_{1}-\varepsilon_{1} \varepsilon} \mathrm{P} \nabla f \mathrm{P}^{2} .
\end{aligned}
$$

From the above, we have

$$
\begin{aligned}
& \frac{d}{d t}\left[\mathrm{P} \nabla v \mathrm{P}^{2}+\left(\varepsilon_{1} \varepsilon+\phi\left(\mathrm{P} \nabla u \mathrm{P}^{2}\right)\right) \mathrm{P} \Delta u \mathrm{P}^{2}\right]+\left(\frac{\varepsilon_{1}-\varepsilon_{1} \varepsilon-8 \varepsilon \mu_{2}-4 \mu_{2} \varepsilon^{2}}{4 \mu_{2}}\right) \mathrm{P} \nabla v \mathrm{P}^{2} \\
& +\left[2 \varepsilon \phi\left(\mathrm{P} \nabla u \mathrm{P}^{2}\right)-\frac{d}{d t}\left(\phi\left(\mathrm{P} \nabla u \mathrm{P}^{2}\right)\right)-\frac{2 \varepsilon^{2}}{\mu_{1}}-2 \varepsilon_{1} \varepsilon\right] \mathrm{P} \Delta u \mathrm{P}^{2} \leq C .
\end{aligned}
$$

Similar to lemma 1.1 formulas (2.14)-(2.19) we can obtain that

$$
\varepsilon_{1} \varepsilon \leq \phi\left(\mathrm{P} \nabla u \mathrm{P}^{2}\right) \leq \frac{\kappa_{1}}{M-2 \varepsilon}\left(1+\kappa_{2} e^{-(M-2 \varepsilon) t}\right) .
$$

where $\varepsilon_{1}, \varepsilon, M, \kappa_{1}, \kappa_{2}$ is constants.

At last, we take proper $\varepsilon, \varepsilon_{1}$, such that: 


$$
\left\{\begin{array}{l}
b_{1}=\frac{\varepsilon_{1}-\varepsilon_{1} \varepsilon-8 \varepsilon \mu_{2}-4 \mu_{2} \varepsilon^{2}}{4 \mu_{2}} \geq 0 \\
b_{2}=2 \varepsilon \phi\left(\mathrm{P} \nabla u \mathrm{P}^{2}\right)-\frac{d}{d t}\left(\phi\left(\mathrm{P} \nabla u \mathrm{P}^{2}\right)\right)-\frac{2 \varepsilon^{2}}{\mu_{1}}-2 \varepsilon_{1} \varepsilon \geq 0 .
\end{array}\right.
$$

Taking $\alpha_{2}=\min \left\{b_{1}, \frac{b_{2}}{\varepsilon_{1} \varepsilon+\phi\left(\mathrm{P} \nabla u \mathrm{P}^{2}\right)}\right\}$, then

$$
\frac{d}{d t} U(t)+\alpha_{2} U(t) \leq C
$$

Where $U(t)=\mathrm{P} \nabla v \mathrm{P}^{2}+\left(\varepsilon_{1} \varepsilon+\phi\left(\mathrm{P} \nabla u \mathrm{P}^{2}\right)\right) \mathrm{P} \Delta u \mathrm{P}^{2}$, by using Gronwall's inequality, we obtain:

$$
U(t) \leq U(0) e^{-\alpha_{2} t}+\frac{C}{\alpha_{2}}\left(1-e^{-\alpha_{2} t}\right) .
$$

Let $0<L=\min \left\{1, \varepsilon_{1} \varepsilon+\phi\left(\mathrm{P} \nabla u \mathrm{P}^{2}\right)\right\}$, so we get

$$
\mathrm{P}(u, v) \mathrm{P}_{\mathrm{H}_{2} \times H_{0}^{1}}^{2}=\mathrm{P} \Delta u \mathrm{P}^{2}+\mathrm{P} \nabla v \mathrm{P}^{2} \leq \frac{U(0)}{L} e^{-\alpha_{2} t}+\frac{C}{\alpha_{2} L}\left(1-e^{-\alpha_{2} t}\right) .
$$

where $v=u_{t}+\varepsilon u$, and $U(0)=\mathrm{P} \nabla v_{0} \mathrm{P}^{2}+\left(\varepsilon_{1} \varepsilon+\phi\left(\mathrm{P} \nabla u_{0} \mathrm{P}^{2}\right)\right) \mathrm{P} \Delta u_{0} \mathrm{P}^{2}, v_{0}=u_{1}+\varepsilon u_{0}$, then

$$
\varlimsup_{t \rightarrow \infty} \mathrm{P}(u, v) \mathrm{P}_{\mathrm{H}_{2} \times H_{0}^{1}}^{2} \leq \frac{C}{\alpha_{2} L} .
$$

So, there exist $R_{1}$ and $t_{2}=t_{2}(\Omega)>0$, such that

$$
\mathrm{P}(u, v) \mathrm{P}_{\mathrm{H}_{2} \times H_{0}^{1}}^{2}=\mathrm{P} \Delta u \mathrm{P}^{2}+\mathrm{P} \nabla v \mathrm{P}^{2} \leq R_{1}\left(t>t_{2}\right) .
$$

\section{Global attractor}

\subsection{The existence and uniqueness of solution}

Theorem 3.1 Assume $\left(G_{1}\right),\left(G_{2}\right)$ hold, let

$$
\begin{gathered}
\left\{\begin{array}{c}
p \geq 2, \quad n=1,2 ; \\
2<p<\frac{n+4}{n}, \quad n \geq 3 .
\end{array}\right. \\
\left\{\begin{array}{cc}
q \geq 2, & n=1,2 ; \\
2<q<\min \left\{\frac{n+4}{n}, \frac{n+2}{n-2}\right\}, & n \geq 3 .
\end{array}\right.
\end{gathered}
$$

and $\mathrm{H}_{2}(\Omega):=H^{2}(\Omega) \cap H_{0}^{1}(\Omega), \quad\left(u_{0}, u_{1}\right) \in \mathrm{H}_{2}(\Omega) \times H_{0}^{1}(\Omega), \quad f \in H_{0}^{1}(\Omega), \quad v=u_{t}+\varepsilon u$, so Equation (1.1) exists a unique smooth solution

$$
(u, v) \in L^{\infty}\left([0,+\infty), \mathrm{H}_{2}(\Omega) \times H_{0}^{1}(\Omega)\right) .
$$

Proof. By the Galerkin method, Lemma 1 and Lemma 2, we can easily obtain the existence of Solutions. Next, we prove the uniqueness of Solutions in detail.

Assume $u, v$ are two solutions of the problems (1.1)-(1.3), let $w=u-v$, then $w(x, 0)=w_{0}(x)=0, w_{t}(x, 0)=w_{1}(x)=0$ and the two equations subtract and obtain 


$$
\begin{aligned}
& w_{t t}-\varepsilon_{1} \Delta w_{t}+\alpha\left(\left|u_{t}\right|^{p-1} u_{t}-\left|v_{t}\right|^{p-1} v_{t}\right)+\beta\left(|u|^{q-1} u-|v|^{q-1} v\right)+ \\
& \left(\phi\left(\mathrm{P} \nabla v \mathrm{P}^{2}\right) \Delta v-\phi\left(\mathrm{P} \nabla u \mathrm{P}^{2}\right) \Delta u\right)=0 .
\end{aligned}
$$

By multiplying (3.2) by $w_{t}$, we get

$$
\begin{aligned}
& \left(w_{t t}-\varepsilon_{1} \Delta w_{t}+\alpha\left(\left|u_{t}\right|^{p-1} u_{t}-\left|v_{t}\right|^{p-1} v_{t}\right)+\beta\left(|u|^{q-1} u-|v|^{q-1} v\right)+\right. \\
& \left.\left(\phi\left(\mathrm{P} \nabla v \mathrm{P}^{2}\right) \Delta v-\phi\left(\mathrm{P} \nabla u \mathrm{P}^{2}\right) \Delta u\right), w_{t}\right)=0 .
\end{aligned}
$$

By using $\mathrm{H} \ddot{O}$ Ider's inequality, Young's inequality and Poincar $e^{\prime}$ 's inequality.

One by one as follows:

$$
\begin{aligned}
& \left(w_{t t}, w_{t}\right)=\frac{1}{2} \frac{d}{d t} \mathrm{P} w_{t} \mathrm{P}^{2} . \\
& \left(-\varepsilon_{1} \Delta w_{t}, w_{t}\right)=\varepsilon_{1} \mathrm{P} \nabla w_{t} \mathrm{P}^{2} \geq \varepsilon_{1} \lambda_{1} \mathrm{P} w_{t} \mathrm{P}^{2} \\
& \left(\alpha\left(\left|u_{t}\right|^{p-1} u_{t}-\left|v_{t}\right|^{p-1} v_{t}\right), w_{t}\right) \\
& =\alpha \int_{\Omega}\left(\left|u_{t}\right|^{p-1} u_{t}-\left|v_{t}\right|^{p-1} v_{t}\right) w_{t} d x \\
& \leq \alpha p \int_{\Omega}\left(\left|u_{t}\right|^{p-1}+\left|v_{t}\right|^{p-1}\right)\left|w_{t} \| w_{t}\right| d x
\end{aligned}
$$

According to Lemma 1 , So, there exists $C_{0}>0$, such that

$$
\begin{aligned}
& \left(\alpha\left(\left|u_{t}\right|^{p-1} u_{t}-\left|v_{t}\right|^{p-1} v_{t}\right), w_{t}\right) \leq \alpha p C_{0} \mathrm{P}_{w_{t}} \mathrm{P}^{2} \\
& \left(\beta\left(|u|^{q-1} u-|v|^{q-1} v\right), w_{t}\right) \\
& \quad=\beta \int_{\Omega}\left(|u|^{q-1} u-|v|^{q-1} v\right) w_{t} d x \\
& \quad \leq \beta q \int_{\Omega}\left(|u|^{q-1}+|v|^{q-1}\right)\left|w \| w_{t}\right| d x
\end{aligned}
$$

According to Lemma 1 , So, there exists $C_{1}>0$, such that

$$
\begin{aligned}
& \left(\beta\left(|u|^{q-1} u-|v|^{q-1} v\right), w_{t}\right) \\
& \leq \beta q C_{1} \mathrm{P} w \mathrm{PP} w_{t} \mathrm{P} \\
& \leq \frac{\varepsilon_{1} \lambda_{1}}{2} \mathrm{P} w_{t} \mathrm{P}^{2}+\frac{\beta^{2} q^{2} C_{1}^{2}}{2 \varepsilon_{1} \lambda_{1}} \mathrm{P} w \mathrm{P}^{2} . \\
& \left(\phi\left(\mathrm{P} \nabla v \mathrm{P}^{2}\right) \Delta v-\phi\left(\mathrm{P} \nabla u \mathrm{P}^{2}\right) \Delta u, w_{t}\right) \\
& \left.=\phi\left(\mathrm{P} \nabla v \mathrm{P}^{2}\right) \Delta v-\phi\left(\mathrm{P} \nabla v \mathrm{P}^{2}\right) \Delta u+\phi\left(\mathrm{P} \nabla v \mathrm{P}^{2}\right) \Delta u-\phi\left(\mathrm{P} \nabla u \mathrm{P}^{2}\right) \Delta u, w_{t}\right) \\
& =\phi\left(\mathrm{P} \nabla v \mathrm{P}^{2}\right)\left(-\Delta w, w_{t}\right)+\left[\phi\left(\mathrm{P} \nabla v \mathrm{P}^{2}\right)-\phi\left(\mathrm{P} \nabla u \mathrm{P}^{2}\right)\right]\left(\Delta u, w_{t}\right) \\
& =\phi\left(\mathrm{P} \nabla v \mathrm{P}^{2}\right) \frac{1}{2} \frac{d}{d t} \mathrm{P} \nabla w \mathrm{P}^{2}+\phi^{\prime}(\xi)(\mathrm{P} \nabla v \mathrm{P}+\mathrm{P} \nabla u \mathrm{P})(\mathrm{P} \nabla v \mathrm{P}-\mathrm{P} \nabla u \mathrm{P})\left(\Delta u, w_{t}\right)
\end{aligned}
$$

where

$$
\begin{aligned}
& \phi^{\prime}(\xi)(\mathrm{P} \nabla v \mathrm{P}+\mathrm{P} \nabla u \mathrm{P})(\mathrm{P} \nabla v \mathrm{P}-\mathrm{P} \nabla u \mathrm{P})\left(\Delta u, w_{t}\right) \\
& \leq\left|\phi^{\prime}(\xi)\right|(\mathrm{P} \nabla v \mathrm{P}+\mathrm{P} \nabla u \mathrm{P})(\mathrm{P} \nabla v-\nabla u \mathrm{P}) \mathrm{P} \Delta u \mathrm{PP} w_{t} \mathrm{P} \\
& \leq \mathrm{P} \phi^{\prime}(\xi) \mathrm{P}_{\infty}(\mathrm{P} \nabla v \mathrm{P}+\mathrm{P} \nabla u \mathrm{P}) \mathrm{P} \Delta u \mathrm{PP} \nabla w \mathrm{PP} w_{t} \mathrm{P} .
\end{aligned}
$$


According to Lemma 1 and Lemma 2, So, there exists $C_{2}>0$, such that

$$
\begin{aligned}
& \phi^{\prime}(\xi)(\mathrm{P} \nabla v \mathrm{P}+\mathrm{P} \nabla u \mathrm{P})(\mathrm{P} \nabla v \mathrm{P}-\mathrm{P} \nabla u \mathrm{P})\left(\Delta u, w_{t}\right) \\
& \leq C_{2} \mathrm{P} \nabla w \mathrm{PP} w_{t} \mathrm{P} \\
& \leq \frac{\varepsilon_{1} \lambda_{1}}{2} \mathrm{P}_{w_{t}} \mathrm{P}^{2}+\frac{C_{2}^{2}}{2 \varepsilon_{1} \lambda_{1}} \mathrm{P} \nabla w \mathrm{P}^{2}
\end{aligned}
$$

Next, according to the basic assumption of Lemma 1 and Lemma 2:

$$
\varepsilon_{1} \varepsilon \leq \phi\left(\mathrm{P} \nabla u \mathrm{P}^{2}\right) \leq \min \left\{\frac{\gamma_{1}}{K-2 \varepsilon}\left(1+\gamma_{2} e^{-(K-2 \varepsilon) t}\right), \frac{\kappa_{1}}{M-2 \varepsilon}\left(1+\kappa_{2} e^{-(M-2 \varepsilon) t}\right)\right\} .
$$

Then, we have

$$
\begin{aligned}
& \left(\phi\left(\mathrm{P} \nabla v \mathrm{P}^{2}\right) \Delta v-\phi\left(\mathrm{P} \nabla u \mathrm{P}^{2}\right) \Delta u, w_{t}\right) \\
& \geq \frac{\varepsilon_{1} \varepsilon}{2} \frac{d}{d t} \mathrm{P} \nabla w \mathrm{P}^{2}-\frac{\varepsilon_{1} \lambda_{1}}{2} \mathrm{P}_{w_{t}} \mathrm{P}^{2}-\frac{C_{2}^{2}}{2 \varepsilon_{1} \lambda_{1}} \mathrm{P} \nabla w \mathrm{P}^{2} .
\end{aligned}
$$

From the above, we have

$$
\begin{aligned}
& \frac{d}{d t}\left[\mathrm{P}_{w_{t}} \mathrm{P}^{2}+\varepsilon_{1} \varepsilon \mathrm{P} \nabla w \mathrm{P}^{2}\right] \\
& \leq 2 \alpha p C_{0} \mathrm{P}_{w_{t}} \mathrm{P}^{2}+\frac{C_{2}^{2}}{\varepsilon_{1} \lambda_{1}} \mathrm{P} \nabla w \mathrm{P}^{2}+\frac{\beta^{2} q^{2} C_{1}^{2}}{\varepsilon_{1} \lambda_{1}} \mathrm{P}_{w} \mathrm{P}^{2} \\
& \leq 2 \alpha p C_{0} \mathrm{P}_{w_{t}} \mathrm{P}^{2}+\frac{C_{2}^{2}}{\varepsilon_{1} \lambda_{1}} \mathrm{P} \nabla w \mathrm{P}^{2}+\frac{\beta^{2} q^{2} C_{1}^{2}}{\varepsilon_{1} \lambda_{1} \mu_{1}} \mathrm{P} \nabla w \mathrm{P}^{2} \\
& \leq 2 \alpha p C_{0} \mathrm{P}_{w_{t}} \mathrm{P}^{2}+\frac{C_{2}^{2} \mu_{1}+\beta^{2} q^{2} C_{1}^{2}}{\varepsilon_{1} \lambda_{1} \mu_{1}} \mathrm{P} \nabla w \mathrm{P}^{2} .
\end{aligned}
$$

Taking $\mathrm{M}=\max \left\{2 \alpha p C_{0}, \frac{C_{2}^{2} \mu_{1}+\beta^{2} q^{2} C_{1}^{2}}{\varepsilon_{1}^{2} \varepsilon \lambda_{1} \mu_{1}}\right\}$, then

$$
\frac{d}{d t}\left(\mathrm{P}_{w_{t}} \mathrm{P}^{2}+\varepsilon_{1} \varepsilon \mathrm{P} \nabla w \mathrm{P}^{2}\right) \leq M\left(\mathrm{P}_{w_{t}} \mathrm{P}^{2}+\varepsilon_{1} \varepsilon \mathrm{P} \nabla w \mathrm{P}^{2}\right)
$$

By using Gronwall's inequality, we obtain

$$
\mathrm{P}_{w_{t}} \mathrm{P}^{2}+\varepsilon_{1} \varepsilon \mathrm{P} \nabla w \mathrm{P}^{2} \leq\left(\mathrm{P}_{w_{t}}(0) \mathrm{P}^{2}+\varepsilon_{1} \varepsilon \mathrm{P} \nabla w(0) \mathrm{P}^{2}\right) \mathrm{e}^{M t} .
$$

So, we can get $\mathrm{P} w_{t} \mathrm{P}^{2}+\varepsilon_{1} \varepsilon \mathrm{P} \nabla w \mathrm{P}^{2} \leq 0$, because of $w_{0}(x)=0, w_{1}(x)=0$.

That shows that

$$
\mathrm{P}_{w} \mathrm{P}^{2}=0, \quad \mathrm{P} \nabla w \mathrm{P}^{2}=0 .
$$

That is

$$
w(x, t)=0
$$

Therefore

$$
u=v \text {. }
$$

So we get the uniqueness of the solution.

Remark 3.1. Under the assumptions of Lemma 1, Lemma 2 and Theorem 3.1, We claim that $S(t)\left(u_{0}, u_{1}\right)=\left(u(t), u_{t}(t)\right)$. Then $S(t)$ composes a continuous semigroup in $\mathrm{H}_{2}(\Omega) \times H_{0}^{1}(\Omega)$. 


\subsection{Global attractor}

Theorem $3.2^{[1,2]}$ Let $E$ be a Banach space, and $\{S(t)\}(t \geq 0)$ are the semigroup operator on $\mathrm{E}$. $S(t): E \rightarrow E, S(t+\tau)=S(t) S(\tau)(\forall t, \tau \geq 0), S(0)=I$, where I is a unit operator. The semigroup operator $S(t)$ satisfies the following conditions.

1) $S(t)$ is uniformly bounded, namely $\forall R>0, \mathrm{P} u \mathrm{P}_{E} \leq R$, it exists a constant $C(R)$, so that

$$
\mathrm{PS}(t) u \mathrm{P}_{E} \leq C(R)(t \in[0,+\infty)) ;
$$

2) It exists a bounded absorbing set $B_{0} \subset E$, namely, $\forall B \subset E$, it exists a constant $t_{0}$, so that

$$
S(t) B \subset B_{0}\left(t \geq t_{0}\right)
$$

where $B_{0}$ and $B$ are bounded sets.

3) When $t>0, S(t)$ is a completely continuous operator A.

Therefore, the semigroup operator $\mathrm{S}(\mathrm{t})$ exists a compact global attractor.

Theorem 3.3 Under the assume of Lemma 1, Lemma 2 and Theorem 3.1, equations have global attractor

$$
\mathrm{A}=\omega\left(B_{0}\right)=\bigcap_{\tau \geq 0} \overline{\bigcup_{t \geq \tau} S(t) B_{0}}
$$

where $\quad B_{0}=\left\{(u, v) \in \mathrm{H}_{2}(\Omega) \times H_{0}^{1}(\Omega): \mathrm{P}(u, v) \mathrm{P}_{\mathrm{H}_{2} \times H_{0}^{1}}^{2}={\mathrm{P} u \mathrm{P}_{2}}_{\mathrm{H}_{2}}^{2}+\mathrm{P} v_{H_{0}^{1}}^{2} \leq R_{0}+R_{1}\right\}, B_{0} \quad$ is $\quad$ the bounded absorbing set of $\mathrm{H}_{2} \times H_{0}^{1}$ and satisfies

1) $S(t) \mathrm{A}=\mathrm{A}, t>0$;

2) $\lim _{t \rightarrow \infty} \operatorname{dist}(S(t) B, \mathrm{~A})=0$, here $B \subset \mathrm{H}_{2} \times H_{0}^{1}$ and it is a bounded set,

$$
\operatorname{dist}(S(t) B, \mathrm{~A})=\sup _{x \in B}\left(\inf _{y \in \mathrm{A}} \mathrm{P} S(t) x-y \mathrm{P}_{\mathrm{H}_{2} \times H_{0}^{1}}\right) \rightarrow 0, t \rightarrow \infty .
$$

Proof. Under the conditions of Theorem 3.1, it exists the solution semigroup $\mathrm{S}(\mathrm{t}), \mathrm{S}(t): \mathrm{H}_{2} \times H_{0}^{1} \rightarrow \mathrm{H}_{2} \times H_{0}^{1}$, here $E=\mathrm{H}_{2}(\Omega) \times H_{0}^{1}(\Omega)$.

(1) From Lemma 1 to Lemma 2, we can get that $\forall B \subset \mathrm{H}_{2}(\Omega) \times H_{0}^{1}(\Omega)$ is a bounded set that includes in the ball $\left\{\mathrm{P}(u, v) \mathrm{P}_{\mathrm{H}_{2} \times H_{0}^{1}} \leq R\right\}$,

$$
\begin{aligned}
& \mathrm{PS}(t)\left(u_{0}, v_{0}\right) \mathrm{P}_{\mathrm{H}_{2} \times H_{0}^{1}}^{2}=\mathrm{P} u \mathrm{P}_{\mathrm{H}_{2}}^{2}+\mathrm{P} v \mathrm{P}_{H_{0}^{1}}^{2} \leq \mathrm{P} u_{0} \mathrm{P}_{\mathrm{H}_{2}}^{2}+\mathrm{P} v_{0} \mathrm{P}_{H_{0}^{1}}^{2}+C \leq R^{2}+C, \\
& \left(t \geq 0,\left(u_{0}, v_{0}\right) \in B\right) .
\end{aligned}
$$

This shows that $S(t)(t \geq 0)$ is uniformly bounded in $\mathrm{H}_{2}(\Omega) \times H_{0}^{1}(\Omega)$.

(2) Furthermore, for any $\left(u_{0}, v_{0}\right) \in \mathrm{H}_{2}(\Omega) \times H_{0}^{1}(\Omega)$, when $t \geq \max \left\{t_{1}, t_{2}\right\}$, we have

$$
\mathrm{PS}(t)\left(u_{0}, v_{0}\right) \mathrm{P}_{\mathrm{H}_{2} \times H_{0}^{1}}^{2}=\mathrm{P} u \mathrm{P}_{\mathrm{H}_{2}}^{2}+\mathrm{P}_{v} \mathrm{P}_{H_{0}^{1}}^{2} \leq R_{0}+R_{1} .
$$

So we get $B_{0}$ is the bounded absorbing set.

(3) Since $E_{1}:=\mathrm{H}_{2}(\Omega) \times H_{0}^{1}(\Omega)^{\circ} E_{0}:=\mathrm{H}_{2}(\Omega) \times L^{2}(\Omega)$ is compact embedded, which means that the 
bounded set in $E_{1}$ is the compact set in $E_{0}$, so the semigroup operator $\mathrm{S}(\mathrm{t})$ exists a compact global attractor $\mathrm{A}$. Furthermore we can know, the global attractor $\mathrm{A}$ is $\omega$-limited set of the absorptive set $B_{0}$, $A=\omega\left(B_{0}\right)=\bigcap_{\tau \geq 0} \overline{\bigcup_{t \geq \tau} S(t) B_{0}}$.

\section{The estimates of the upper bounds of Hausdorff and fractal dimensions for the global attractor}

In order to obtain an estimate of the upper bounds of Hausdorff and fractal dimensions for the global attractor $\mathrm{A}$ of the problems (1.1)-(1.3). we rewrite the problems (1.1)-(1.3):

$$
\begin{aligned}
& u_{t t}+\varepsilon_{1} A u_{t}+\phi\left(\mathrm{P}^{\frac{1}{2}} u \mathrm{P}^{2}\right) A u+h(u)=f(x) \text { in } \Omega \times \mathrm{R}^{+}, \\
& u(x, 0)=u_{0}(x) ; u_{t}(x, 0)=u_{1}(x), \quad x \in \Omega, \\
& \left.u(x, t)\right|_{\partial \Omega}=0,\left.A u(x, t)\right|_{\partial \Omega}=0, \quad x \in \Omega .
\end{aligned}
$$

Let $\mathrm{Au}=-\Delta u, h(u)=\alpha\left|u_{t}\right|^{p-1} u_{t}+\beta|u|^{q-1} u$, where $\Omega$ is a bounded domain in $\mathrm{R}^{N}$ with smooth boundary $\partial \Omega$, and $\varepsilon_{1}, \alpha, \beta$ are positive constants. We consider the abstract linearized equations of the above equations as follows:

$$
\begin{aligned}
& U_{t}+A U=F U, \\
& U_{0}=\xi, U_{t}(0)=\zeta .
\end{aligned}
$$

Let $U_{0} \in H_{0}^{1}(\Omega), U(t)$ is the solution of the problems (4.20)-(4.21). We can prove that the problems (4.20)-(4.21) have a unique solution $U \in L^{\infty}\left(0, T, H_{0}^{1}(\Omega)\right), U_{t} \in L^{\infty}\left(0, T, L^{2}(\Omega)\right)$. The equation (4.20) is the linearized equation by the equation (4.17).

Define the mapping $L s(t)_{u_{0}}: L s(t)_{u_{0}} \zeta=U(t)$.

Let

$$
u(t)=s(t) u_{0}
$$$$
\varphi_{0}=\left(u_{0}, u_{1}\right), \overline{\varphi_{0}}=\varphi_{0}+\{\xi, \zeta\}=\left\{u_{0}+\xi, u_{1}+\zeta\right\}
$$

$\mathrm{P} \varphi_{0} \mathrm{P}_{E_{0}} \leq R_{1}, \mathrm{P} \overline{\varphi_{0}} \mathrm{P}_{E_{0}} \leq R_{2}, E_{0}=\mathrm{V} \times \mathrm{H}, \mathrm{V}:=H_{0}^{1}(\Omega), \mathrm{H}:=L^{2}(\Omega) ; S(t) \varphi_{0}=\varphi(t)=\left\{u(t), u_{t}(t)\right\}, S(t) \overline{\varphi_{0}}=\left\{\varphi(t), \overline{\varphi_{t}}(t)\right\}$

Lemma 4.1 ${ }^{[11]}$ Assume $\mathrm{H}$ is a Hilbert space, $E_{0}$ is a compact set of $\mathrm{H}$.

$S(t): E_{0} \rightarrow H$ is a continuous mapping,satisfy the follow conditions.

1) $S(t) E_{0}=E_{0}, t>0$;

2) If $S(t)$ is $\operatorname{Fr} e^{\prime}$ chet differentiable, it exists a bounded linear differential operator $L\left(t, \varphi_{0}\right) \in C\left(R^{+} ; L\left(E_{0}, E_{0}\right)\right), \forall t>0$, that is

$$
\frac{\mathrm{PS}(t) \overline{\varphi_{0}}-S(t) \varphi_{0}-L\left(t, \varphi_{0}\right)(u, v) \mathrm{P}_{E_{0}}^{2}}{\mathrm{P}\{\xi, \zeta\} \mathrm{P}_{E_{0}}^{2}} \rightarrow 0,\{\xi, \zeta\} \rightarrow 0
$$

where $L\left(t, \varphi_{0}\right):\{\xi, \zeta\} \rightarrow\left\{U(t), U_{t}(t)\right\} . U(t)$ is the solution of problems (4.20)-(4.21).

The proof of lemma 4.1 see ref. [11], is omitted here.

According to Lemma 4.1, we can get the following theorem :

Theorem 4.1 ${ }^{[11,12]}$ Let $A$ is the global attractor that we obtain in section 3 . In that case, $A$ has finite Hausdorff dimensions and fractal dimensions in $\left(H_{2}(\Omega) \cap H_{0}^{1}(\Omega)\right) \times H_{0}^{1}(\Omega)$, that is $d_{H}(\mathrm{~A}) \leq n, d_{F}(\mathrm{~A}) \leq \frac{8 n}{7}$. 
Proof. Firstly, we rewrite the equations $(4.17),(4.18)$ into the first order abstract evolution equations in $E_{0}$.

Let $\Psi=R_{\varepsilon} \varphi=\left\{u, u_{t}+\varepsilon u\right\}, R_{\varepsilon}:\left\{u, u_{t}\right\} \rightarrow\left\{u, u_{t}+\varepsilon u\right\}$, is an isomorphic mapping. So let $\mathrm{A}$ is the global attractor of $\{S(t)\}$, then $R_{\varepsilon} \mathrm{A}$ is also the global attractor of $\left\{S_{\varepsilon}(t)\right\}$, and they have the same dimensions. $0<\varepsilon \leq \varepsilon_{0}, \varepsilon_{0}=\min \left\{\frac{\varepsilon_{1}}{4}, \frac{\lambda_{1}}{2 \varepsilon_{1}}\right\}$, then $\Psi$ satisfies as follows:

$$
\begin{aligned}
& \Psi_{t}+\Lambda_{\varepsilon} \Psi+\bar{h}(\Psi)=\bar{f}, \\
& \Psi(0)=\left\{u_{0}, u_{1}+\varepsilon u_{0}\right\}^{T} .
\end{aligned}
$$

where $\Psi=\left\{u, u_{t}+\varepsilon u\right\}^{T}, \bar{h}(\Psi)=\{0, h(u)\}^{T}, \bar{f}=\{0, f(x)\}^{T}$,

$$
\begin{gathered}
\Lambda_{\varepsilon}=\left(\begin{array}{cc}
\varepsilon I & -I \\
\phi\left(\mathrm{P}^{\frac{1}{2}} \mathrm{P}^{2}\right) A-\varepsilon_{1} \varepsilon A & \varepsilon_{1} A
\end{array}\right) \\
\Psi_{t}:=F(\Psi)=\bar{f}-\Lambda_{\varepsilon} \Psi-\bar{h}(\Psi) . \\
P_{t}=F_{t}(\Psi) . \\
P_{t}+\Lambda_{\varepsilon} P+\bar{h}_{t}(\Psi) P=0 .
\end{gathered}
$$

where $P=\left\{U, U_{t}+\varepsilon U\right\}^{T}, \bar{h}_{t}(\Psi) P=\left\{0, h_{t}(u) U\right\}^{T}$. The initial condition (4.21) can be written in the following form:

$$
P(0)=\omega, \omega=\{\xi, \zeta\} \in E_{0} .
$$

We take $n \in N$, then consider the corresponding $\mathrm{n}$ solutions: $\left(P=P_{1}, P_{2}, \ldots, P_{n} . P_{j} \in E_{0}\right)$ of the initial values: $\left(\omega=\omega_{1}, \omega_{2}, \ldots, \omega_{n} . \omega_{j} \in E_{0}\right)$ in the equations (4.26)-(4.28). So there is

$$
\left|P_{1}(t) \wedge P_{2}(t) \wedge \ldots \wedge P_{n}(t)\right|_{\wedge_{E_{0}}^{n}}=\left|\omega_{1} \wedge \omega_{2} \wedge \ldots \wedge \omega_{n}\right|_{\wedge_{E_{0}}^{n}} \cdot e^{\int_{0}^{t} \operatorname{Tr} F_{t}\left(S_{\varepsilon}(\tau) \Psi_{0}\right) \cdot Q_{n}(\tau) d \tau}
$$

from $\quad \psi(\tau)=S_{\varepsilon}(\tau) \Psi_{0}, \quad$ we $\quad$ get $\quad S_{\varepsilon}(\tau):\left\{u_{0}, v_{1}=u_{1}+\varepsilon u_{0}\right\} \rightarrow\left\{u(\tau), v(\tau)=u_{t}(\tau)+\varepsilon u(\tau)\right\}$, $\psi(\tau)=\left\{u(\tau), v_{t}(\tau)=u_{t}(\tau)+\varepsilon u(\tau)\right\}$

Here $\mathrm{u}$ is the solution of the problems $(4.17)-(4.19) ; \wedge$ represents the outer product, Tr represents the trace, $Q_{n}(\tau)=Q_{n}\left(\tau, \Psi_{0} ; \omega_{1}, \omega_{2}, \ldots, \omega_{n}\right)$ is an orthogonal projection from the space $E_{0}=\mathrm{V} \times \mathrm{H}$ to the subspace spanned by $\left\{P_{1}(\tau), P_{2}(\tau), \ldots, P_{n}(\tau)\right\}$.

For a given time $\tau$, let $\phi_{j}(\tau)=\left\{\xi_{j}(\tau), \zeta_{j}(\tau)\right\}, j=1,2, \ldots, n .\left\{\phi_{j}(\tau)\right\}_{j=1,2, \ldots n}$ is the standard orthogonal basis of the space $Q_{n}(\tau)_{E_{0}}=\operatorname{span}\left[P_{1}(\tau), P_{2}(\tau), \ldots, P_{n}(\tau)\right]$.

From the above, we have

$$
\begin{aligned}
\operatorname{Tr}_{t}(\Psi(\tau)) \cdot Q_{n}(\tau) & \left.=\sum_{j=1}^{\infty} F_{t}(\Psi(\tau)) \cdot Q_{n}(\tau) \phi_{j}(\tau), \phi_{j}(\tau)\right)_{E_{0}} \\
& \left.=\sum_{j=1}^{n} F_{t}(\Psi(\tau)) \phi_{j}(\tau), \phi_{j}(\tau)\right)_{E_{0}}
\end{aligned}
$$


where $(\cdot,)_{E_{0}}$ is the inner product in $E_{0}$. Then

$(\{\xi, \zeta\},\{\bar{\xi}, \bar{\zeta}\})_{E_{0}}=(\xi, \bar{\xi})+(\zeta, \bar{\zeta}) ;\left(F_{t}(\Psi) \phi_{j}, \phi_{j}\right)_{E_{0}}=-\left(\Lambda_{\varepsilon} \phi_{j}, \phi_{j}\right)_{E_{0}}-\left(h_{t}(u) \xi_{j}, \xi_{j}\right)$;

Let $A \zeta_{j}=\lambda_{j} \zeta_{j}$, here $\lambda_{j}$ is the eigenvalue of characteristic vector $\zeta_{j}$ about A. Using the method similar to the priori estimates in Lemma 1 and Lemma 2 to obtain:

$$
\begin{aligned}
\left(\Lambda_{\varepsilon} \phi_{j}, \phi_{j}\right)_{E_{0}} & =\varepsilon \mathrm{P} \xi_{j} \mathrm{P}^{2}+\left(\phi-\varepsilon_{1} \varepsilon\right)\left(A \zeta_{j}, \xi_{j}\right)-\left(\xi_{j}, \zeta_{j}\right)+\varepsilon_{1}\left(A \zeta_{j}, \zeta_{j}\right) \\
& =\varepsilon \mathrm{P} \xi_{j} \mathrm{P}^{2}+\left(\phi-\varepsilon_{1} \varepsilon\right) \lambda_{j}\left(\zeta_{j}, \xi_{j}\right)-\left(\xi_{j}, \zeta_{j}\right)+\varepsilon_{1} \lambda_{j} \mathrm{P} \zeta_{j} \mathrm{P}^{2} \\
& \geq a\left(\mathrm{P} \xi_{j} \mathrm{P}^{2}+\mathrm{P} \zeta_{j} \mathrm{P}^{2}\right) .
\end{aligned}
$$

where $a:=\min \left\{\frac{2 \varepsilon-l \lambda_{j}-1}{2}, \frac{2 \varepsilon_{j}-l \varepsilon_{j}-1}{2}\right\}$, let $l=\phi-\varepsilon_{1} \varepsilon$

Now, suppose that $\left\{u_{0}, u_{1}\right\} \in \mathrm{A}$, according to theorem 3.3, $\mathrm{A}$ is a bounded absorbing set in $E_{1}$.

$\Psi(t)=\left\{u(t), u_{t}(t)+\varepsilon u(t)\right\} \in E_{1}, u(t) \in D(A) ; D(A)=\{u \in \mathrm{V}, A u \in \mathrm{H}\}$.

Then there is a $s \in[0,1]$ to make the mapping $h_{t}: D(A) \rightarrow \rho\left(\mathrm{V}_{s}, \mathrm{H}\right)$. At the same time, there are the following results:

$$
\begin{aligned}
& R_{A}=\sup _{\{\xi, \zeta\} \in \mathrm{A}}|A \xi|<\infty ; \\
& \sup _{\substack{u \in D(A) \\
|A u|<R_{A}}}\left|h_{t}(u)\right|_{\rho\left(v_{s}, \mathrm{H}\right)} \leq r<\infty .
\end{aligned}
$$

where $\mathrm{P} h_{t}(u) \xi_{j}, \zeta_{j} \mathrm{P}$ meets: $\mathrm{P} h_{t}(u) \xi_{j}, \zeta{ }_{j} \mathrm{P} \leq r \mathrm{P} \xi_{j} \mathrm{P}_{s} \mathrm{P} \zeta_{j} \mathrm{P}$.

Comprehensive above can be obtained:

$$
\begin{aligned}
\left(F_{t}(\Psi) \phi_{j}, \phi_{j}\right)_{E_{0}} & \leq-a\left(\mathrm{P} \xi_{j} \mathrm{P}^{2}+\mathrm{P} \zeta_{j} \mathrm{P}^{2}\right)+r \mathrm{P} \xi_{j} \mathrm{P} P{ }_{s} \mathrm{P} . \\
& \leq-\frac{a}{2}\left(\mathrm{P} \xi_{j} \mathrm{P}^{2}+\mathrm{P} \zeta_{j} \mathrm{P}^{2}\right)+\frac{r^{2}}{2 a} \mathrm{P} \xi_{j} \mathrm{P}_{s}^{2} .
\end{aligned}
$$

$\mathrm{P} \xi_{j} \mathrm{P}^{2}+\mathrm{P} \zeta{ }_{j} \mathrm{P}^{2}=\mathrm{P} \phi_{j} \mathrm{P}_{E_{0}}^{2}=1$, due to $\left\{\phi_{j}(\tau)\right\}_{j=1,2, \ldots, n}$ is a standard orthogonal basis in $Q_{n}(\tau)_{E_{0}}$. So

$$
\left.\sum_{j=1}^{n} F_{t}(\Psi(\tau)) \phi_{j}(\tau), \phi_{j}(\tau)\right)_{E_{0}} \leq-\frac{n a}{2}+\frac{r^{2}}{2 a} \mathrm{P} \xi_{j} \mathrm{P}_{s}^{2}
$$

Almost to all t, making

$$
\sum_{j=1}^{n} \mathrm{P} \xi_{j} \mathrm{P}_{s}^{2} \leq \sum_{j=1}^{n-1} \lambda_{j}^{s-1}
$$

So

$$
\operatorname{Tr}_{t}(\Psi(\tau)) \cdot Q_{n}(\tau) \leq-\frac{n a}{2}+\frac{r^{2}}{2 a} \sum_{j=1}^{n-1} \lambda_{j}^{s-1}
$$

Let us assume that $\left\{u_{0}, u_{1}\right\} \in \mathrm{A}$, is equivalent to $\Psi_{0}=\left\{u_{0}, u_{1}+\varepsilon u_{0}\right\} \in R_{\varepsilon} \mathrm{A}$.

Then 


$$
\begin{aligned}
& q_{n}(t)=\sup _{\Psi_{0} \in R_{\varepsilon} \mathrm{A}} \sup _{\substack{\omega \in E_{0} \\
\mathrm{P}_{\omega \mathrm{P}_{E_{0}}} \leq 1}}\left(\frac{\int_{0}^{t} \operatorname{Tr} F_{t}\left(S_{\varepsilon}(\tau) \Psi_{0}\right) \cdot Q_{n}(\tau) d \tau}{t_{0}}\right) \cdot j=1,2, \ldots, n . \\
& q_{n}=\limsup _{t \rightarrow \infty} q_{n}(t) .
\end{aligned}
$$

According to (4.35),(4.36), so

$$
\begin{aligned}
& q_{n}(t) \leq-\frac{n a}{2}+\frac{r^{2}}{2 a} \sum_{j=1}^{n-1} \lambda_{j}^{s-1}, \\
& q_{n} \leq-\frac{n a}{2}+\frac{r^{2}}{2 a} \sum_{j=1}^{n-1} \lambda_{j}^{s-1} .
\end{aligned}
$$

Therefore, the Lyapunov exponent of $\mathrm{A}\left(\operatorname{or} R_{\varepsilon} \mathrm{A}\right)$ is uniformly bounded.

$$
\mu_{1}+\mu_{2}+\cdots+\mu_{n} \leq-\frac{n a}{2}+\frac{r^{2}}{2 a} \sum_{j=1}^{n} \lambda_{j}^{s-1} .
$$

From (4.36), when $n \rightarrow \infty, q_{n} \rightarrow 0$. By the compactness of the operator $A^{-1}$, we can get further to :When $j \rightarrow \infty, \lambda_{i} \rightarrow \infty$. So, when $n \rightarrow \infty$, such that

$$
\frac{1}{n} \sum_{j=1}^{n} \lambda_{j}^{s-1} \rightarrow 0
$$

From what has been discussed above, it exists $n \geq 1$,a,r are constants,

then

$$
\begin{aligned}
\frac{1}{n} \sum_{j=1}^{n} \lambda_{j}^{s-1} & \leq \frac{a^{2}}{8 r^{2}} . \\
q_{n} & \leq-\frac{n a}{2}\left(1-\frac{r^{2}}{a^{2}} \sum_{j=1}^{n} \lambda_{j}^{s-1}\right) \leq-\frac{7 n a}{16} . \\
\left(q_{j}\right)_{+} & \leq \frac{r^{2}}{2 a} \sum_{i=1}^{j} \lambda_{i}^{s-1} \leq \frac{r^{2}}{2 a} \sum_{i=1}^{j} \lambda_{i}^{s-1} \leq \frac{n a}{16}, j=1,2, \ldots, n .
\end{aligned}
$$

So we finally obtained the following conclusions:

$$
\max _{1 \leq j \leq n-1} \frac{\left(q_{j}\right)_{+}}{\left|q_{n}\right|} \leq \frac{1}{7} .
$$

According to the reference $[11,12]$, we immediately to the Hausdorff dimension and fractal dimension are respectively $d_{H}(\mathrm{~A}) \leq n, d_{F}(\mathrm{~A}) \leq \frac{8 n}{7}$.

\section{Acknowledgements}

The authors express their sincere thanks to the anonymous reviewer for his/her careful reading of the paper, giving valuable comments and suggestions. These contributions greatly improved the paper.This work is supported by the Nature Science Foundation of China(No.11561076) 


\section{References}

1. Lin Guoguang. Nonlinear evolution equation,Yunnan University Press,2011.

2. A.V. Babin and M.I. Vishik. Attractors of Evolution Equations. Studies in Math. and Its Appl. vol.25,New York: North-Holland, 1992.

3. Guoguang Lin; Fangfang Xia and Guigui Xu. The global and pullback attractors for a strongly damped wave equation with delays. International Journal of Modern Nonlinear Theory and Application.2(2013),pp.209-218.

4. Mitsuhiro Nakao. An attractor for a nonlinear dissipative wave equation of Kirchhoff type. Journal of Mathematical Analysis and Applications.353(2009),pp.652-659.

5. Yongqin Xie and Chengkui Zhong. The existence of global attractors for a class nonlinear evolution equation. Journal of Mathematical Analysis and Applications,336(2007),pp.54-69.

6. Kirchhoff,G.: Vorlesungen Uber Mechanik. Teubner,Leipzig(1883).

7. Zhijian Yang; Pengyan Ding and Zhiming Liu. Global attractor for the Kirchhoff type equations with strong nonlinear damping and supercritical nonlinearity. Applied Mathematics Letters.33(2014),pp.12-17.

8. Yang Zhijian and Wang Yunqing. Global attractor for the Kirchhoff type equation with a strong dissipation. Journal of Differential Equations.249(2010),pp.3258-3278.

9. Meixia Wang; Cuicui Tian; Guoguang Lin. Global attractor and dimension estimation for a 2D generalized anisotropy Kuramoto-Sivashinsky equation. International Journal of Modern Nonlinear Theory and Application.3(2014),pp.163-172.

10. Liang Guo; Zhaoqin Yuan and Guoguang Lin. The global attractors for a nonlinear viscoelastic wave equation with strong damping and linear damping and source terms. International Journal of Modern Nonlinear Theory and Application.4(2015),pp.142-152.

11. Teman,R.:Infinite Dimensional Dynamics Systems in Mechanics and Physics.Springer,New York(1998).

12. Wu Jingzhu; Lin Guoguang. The global attractor of the Bossinesq equation with damping term and its dimension estimation. Journal of Yunnan University,31(2009),pp.335-340.

13. Vincent; Xiaosong Liu. A sharp lower bound for the Hausdorff dimension of the globlal attractors of the 2D Navier-Stokes equations. Communications in Mathematical Physics,158(1993),pp.327-339. 